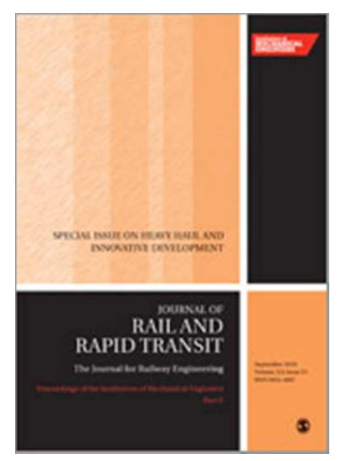

\title{
Effect of Underfloor Wheel Lathe Turning Characteristics on the Evolution of Wheel Polygonisation
}

\begin{tabular}{|r|l|}
\hline Journal: & Part F: Journal of Rail and Rapid Transit \\
\hline Manuscript ID & JRRT-17-0235.R1 \\
\hline Manuscript Type: & Article \\
\hline Complete List of Authors: & $\begin{aligned} \text { Cui, Dabin; Southwest Jiaotong University, school of mechnical engineering } \\
\text { An, Boyang; Southwest Jiaotong University, } \\
\text { Allen, Paul; University of Huddersfield, IRR } \\
\text { Wang, Ruichen; Institute of Railway Research, School of Computing and } \\
\text { Engineering } \\
\text { Wang, Ping; Southwest Jiaotong University } \\
\text { Wen, Zefeng; State Key Laboratory of Traction Power } \\
\text { Li, Li; Southwest Jiaotong University, }\end{aligned}$ \\
\hline Keywords: & $\begin{array}{l}\text { EMU wheels, Underfloor Wheel Lathe, Wheel Polygon, Turning quality, } \\
\text { Friction roller eccentricity }\end{array}$ \\
\hline \multirow{5}{*}{ Abstract: } & $\begin{array}{l}\text { During both running and wheel cut operations, railway vehicle wheels and } \\
\text { the friction rollers which support and drive the wheelset on a typical wheel } \\
\text { cut lathe are subject to wear and hence are likely to develop out-of-round } \\
\text { characteristics after sustained use. The resulting out-of-round wheels can } \\
\text { significantly affect the ride quality of services and can potentially increase } \\
\text { the incidence of fatigue related component failures due to resultant higher } \\
\text { intensity loading cycles. Furthermore, the corresponding out-of-round of } \\
\text { the lathe's friction rollers will continue to degrade subsequent cut quality of } \\
\text { wheels. To allow analysis of the out-of-round characteristics generated } \\
\text { using an underfloor wheel lathe operated by China high Speed train, a } \\
\text { mathematical model based on typical EMU vehicle's wheelsets and their } \\
\text { interactions with the wheel lathe friction rollers was established. The } \\
\text { influencing factors on cut process, including number of cuts, eccentricity } \\
\text { forms of the friction rollers, and the longitudinal spacing of the two rollers } \\
\text { have on the cut quality of the wheels has been analysed. The results show } \\
\text { that two cut can effectively remove the high order polygon on the wheel } \\
\text { surface. The eccentricity and phase angle of the friction rollers has no } \\
\text { influence on the cut quality of high order polygons, whereas conversely, it }\end{array}$ \\
\hline
\end{tabular}


is the primary cause of 4 th order polygons. The severity of the 4 th order polygon depends on the level and the phase of the eccentricity of the friction rollers. The space of the two rollers can also affect the cut quality significantly. The outcomes of this study are the theoretical and practical value for maintenance of polygonised wheels using the underfloor lathe.

SCHOLARONE ${ }^{m}$

Manuscripts 
Effect of Underfloor Wheel Lathe Turning Characteristics on the Evolution of Wheel Polygonisation

\author{
Dabin Cui ${ }^{1,2^{*}}$, Boyang An ${ }^{2}$, Paul Allen ${ }^{3}$, Ruichen Wang ${ }^{3}$, Ping Wang ${ }^{2}$, Zefeng Wen ${ }^{4}$, Li Li $^{1}$ \\ 1 School of Mechanical Engineering, Southwest Jiaotong University, Chengdu 610031, China; \\ 2 Key Laboratory of High-speed Railway Engineering, Ministry of Education, Chengdu 610031, \\ China; \\ 3 Insititute of railway research, University of Huddersfield, Huddersfield HD1 3DH, UK; \\ 4 State Key Laboratory of Traction Power, Southwest Jiaotong University, Chengdu 610031, China;
}

*Corresponding author. Email: cdb1645@163.com. Tel: +86-15982365391. 
Effect of Underfloor Wheel Lathe Turning Characteristics on the Evolution of Wheel Polygonisation

\author{
Dabin Cui ${ }^{1,2}$, Boyang An ${ }^{2}$, Paul Allen ${ }^{3}$, Ruichen Wang ${ }^{3}$, Ping Wang ${ }^{2}$, Zefeng Wen ${ }^{4}, \mathrm{Li} \mathrm{Li}^{{ }^{*}}$ \\ 1. School of Mechanical Engineering, Southwest Jiaotong University, Chengdu 610031, China; \\ 2. Key Laboratory of High-speed Railway Engineering, Ministry of Education, Chengdu 610031, China; \\ 3. Insititute of Railway Research, University of Huddersfield, Huddersfield HD1 3DH, UK; \\ 4. State Key Laboratory of Traction Power, Southwest Jiaotong University, Chengdu 610031, China;
}

\begin{abstract}
During both running and wheel cut operations, railway vehicle wheels and the friction rollers which support and drive the wheelset on a typical wheel cut lathe are subject to wear and hence are likely to develop out-of-round characteristics after sustained use. The resulting out-of-round wheels can significantly affect the ride quality of services and can potentially increase the incidence of fatigue related component failures due to resultant higher intensity loading cycles. Furthermore, the corresponding out-of-round of the lathe's friction rollers will continue to degrade subsequent cut quality of wheels. To allow analysis of the out-of-round characteristics generated using an underfloor wheel lathe operated by China high Speed train, a mathematical model based on typical EMU vehicle's wheelsets and their interactions with the wheel lathe friction rollers was established. The influencing factors on cut process, including number of cuts, eccentricity forms of the friction rollers, and the longitudinal spacing of the two rollers have on the cut quality of the wheels has been analysed. The results show that two cut can effectively remove the high order polygon on the wheel surface. The eccentricity and phase angle of the friction rollers has no influence on the cut quality of high order polygons, whereas conversely, it is the primary cause of $4^{\text {th }}$ order polygons. The severity of the $4^{\text {th }}$ order polygon depends on the level and the phase of the eccentricity of the friction rollers. The space of the two rollers can also affect the cut quality significantly. The outcomes of this study are the theoretical and practical value for maintenance of polygonised wheels using the underfloor lathe.
\end{abstract}

Keywords: EMU wheels, Underfloor wheel lathe, Wheel polygon, Cut quality, Friction roller eccentricity

\title{
1 INTRODUCTION
}

A vehicle's wheels are subject to a demanding loading regime, whereby high contact pressures and tangential forces combine to generate wear of the wheel tread and flange. Once wear levels exceed predetermined maintenance limits the vehicle is required to be removed from service for the wheelsets to be re-profiled or replaced [1-2]. In order to reduce the maintenance cost and raise efficiency, specialised wheel lathes have been designed and developed to re-profile the wheels. Underfloor wheel lathes are highly modernised and specialist equipment specifically designed for the re-profiling of railway wheelsets. They can efficiently renew the wheel profile without requiring the removal of the wheelsets from the 
rolling stock. Most studies on wheel lathe operations focus on manufacturing technologies and control [3-5] whilst few works explore the cut process and wheel defects.

During the evolution of wheel wear, irregularities may develop on the tread surface which when considered on a radial basis take on a polygonised form. Typically it has been found that radial irregularities including 1 to 24 wavelengths around the circumference of the wheel are a persistent problem on high-speed trains in China [6-7]. The N wavelengths means there are $\mathrm{N}$ harmonic waves along the circumference of the wheel. As the size of the irregularities grows, the ride comfort begins to be significantly affected, whilst structural loading is also increased, leading to a potential loss of fatigue life of the vehicle structures. [8-11].

Currently, the wheel tread polygonization cannot be solved as the root cause of the mechanism that is not well understood. The primary mitigation in the removal of problems associated with polyogonisation is to re-profile the wheel using the underfloor wheel lathe. However, underfloor wheel lathes are not typically designed to treat polygonal wheels and hence within a significant proportion of newly turned wheels the polygonal irregularities remain. Therefore effective removal of wheel polygonisation using existing underfloor wheel lathes needs to be assessed to improve maintenance practices and effectiveness.

This paper presents a simulation model to predict the state of wheel polygonisation after the wheelset is turned by a type of underfloor wheel lathe to assess the quality of he re-profiled wheels. Several influencing parameters are considered during the assessment the machining cycles (processing on entire wheel surface), eccentricity forms of the friction rollers, the spacing rollers on one wheel and the wheel polygonization.

\section{Description of the polygonal wheel}

The wear status of the wheel of EMU train and the friction rollers of the underfloor wheelset lathe are investigated. Fig.1 (a) shows the radial deviation from the mean radius of a polygonal wheel and an normal wheel, $r(\theta)=R(\theta)-R_{0}$, measured on EMUs. In this equation, $R_{0}$ is the mean wheel radius and $R(\theta)$ is the radial profile at the corresponding angle $\theta$. The polygonization with $19^{\text {th }}$ order harmonics around the polygonal wheel circumference can be clearly seen in Fig.1 (a). The term radial eccentricity $(D r)$ which represents the difference between the maximum radius and the minimum radius of the wheel is used to denote the out-of-round level. However, this relatively simple description of the wheel does not provide a good indication of the level of polyogonisation. A better description can be achieved by translating the radial deviation in fig.1 (a) into the Cartesian coordinate system. The radial deviation can then be presented as a function $r(x)$ of the circumferential distance $x$ as shown in Fig.1 (b). The roughness level $L_{r}^{k}$ is 
defined by

$$
L_{r}^{k}=10 \log _{10}\left(r_{k}^{2} / r_{r e f}^{2}\right)[\mathrm{dB} \text { re } 1 \mu \mathrm{m}]
$$

Where, $r_{r e f}=1 \mu m \cdot r_{k}^{2}\left[\mathrm{~m}^{2}\right]$ is the mean square value of the roughness profile $r(x)[\mathrm{m}]$ evaluated in one-third octave band $k$ with centre wavelength

$$
\lambda_{k}=0.01 \times 10^{k / 10}[\mathrm{~m}], k=-10,-9, \ldots, 14,15
$$

The Amplitudes for different wavelengths were calculated with the Hilbert-Huang transform [12]. The mean square value $r_{k}^{2}$ is obtained by averaging the squares of the amplitudes of the resulting narrowband spectrum in each one-third octave band, as shown in Fig.1(c). The first order polygonal corresponds to the eccentricity of the wheel, the second to the ovality, etc.

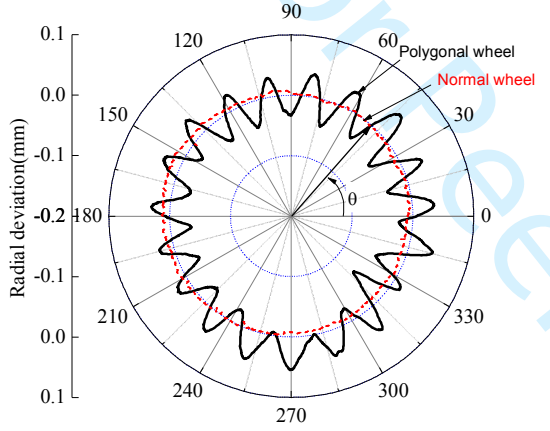

(a) Raw data in Polar coordinate system

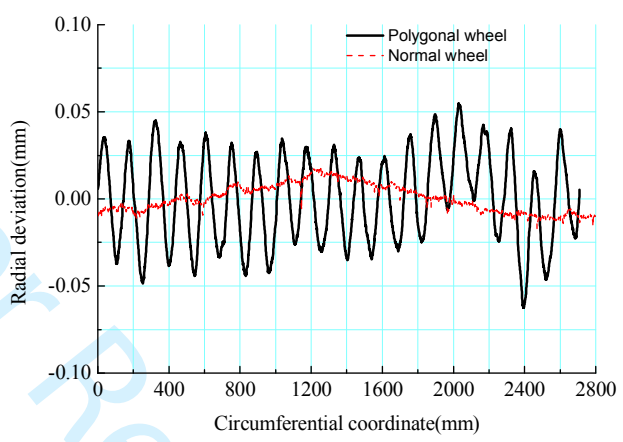

(b)Raw data in Cartesian coordinate system

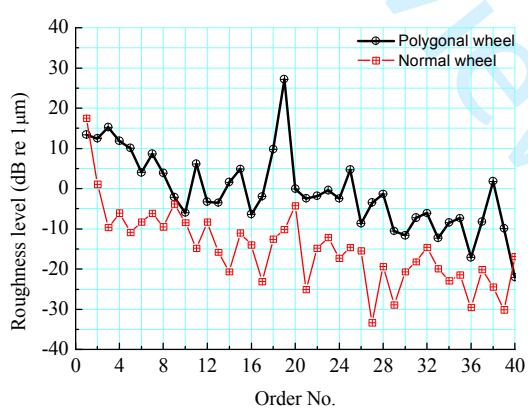

(c) Roughness spectrum

Fig. 1 Examples of the measured polygonal wheel and normal wheel

From Fig. 1 (c) it can be seen that the Polygonal wheel has a high roughness level at the $19^{\text {th }}$ order. This result is typical of many service vehicles measured in China, whereby a large proportion have been found with polygonal wheels dominated by $18^{\text {th }} \sim 20^{\text {th }}$ order polygon. These defects are causing significant in-train vibrations and in some more severe cases evidence of structural damage has been found. 


\section{Wheel lathe roller eccentricity}

An underfloor wheel lathe is typically a very frequently used machine and hence deterioration, primarily in the form of component wear, can begin to create running defects between the lathe's drive rollers and the wheelset being turned. Fig.2 illustrates a type wheel lathe facility.

In the process of wheelset cut, the bogie is positioned on the lathe with the wheelset located between two friction rollers. The clamping devices push the axle boxes down to ensure correct and robust contact with the friction rollers. The friction rollers drive the wheelsets to facilitate the cut process. The actual radius of the turned wheel between wheelset centre and the lathe tools is in fact not a constant value with respect to the wheel rotation due to out-of-round wheel and worn friction rollers, which can lead a poor cut quality. In addition to deviations related to the wheelset, the friction rollers and the driver gears are also subjected to wear. The combination of these factors affects the re-profiling precision of the wheel lathe.

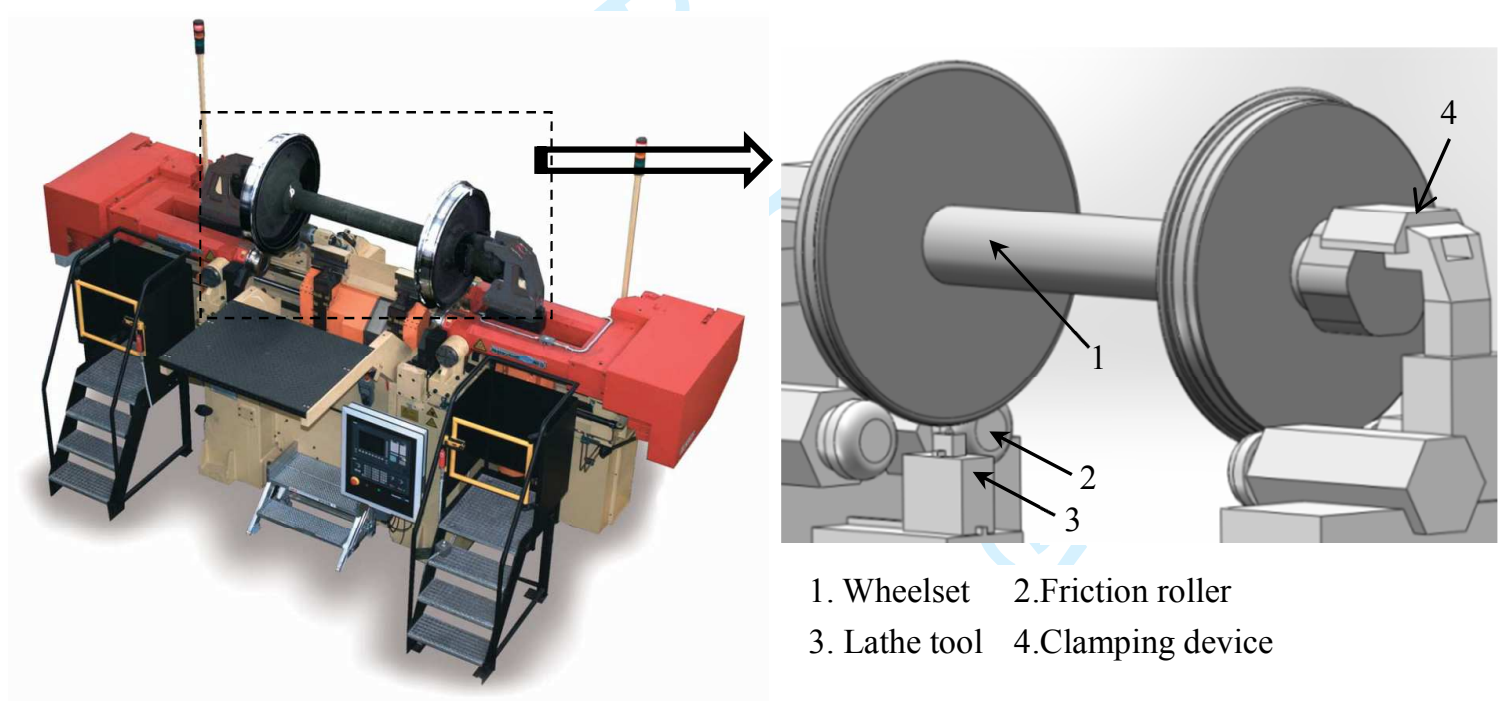

Fig.2 Underfloor wheelset lathe

Based on the 16 measured data of wheel lathes on four main depots in China, the realistic roughness levels of the friction rollers are shown in Fig. 3 as an example. The two friction rollers are symmetrically placed on both sides of the lathe machine with different eccentricity. The calculated maximum values of eccentricity were approximately 24.5 $d B$ re $1 \mu m$ and $15 d B$ re $1 \mu m$, see Fig.3(b). According to the previous research on wheel lathe cut[13], the findings show that the eccentricity of the friction rollers is a key factor in the turning quality of the wheel lathe and the performance of the friction roller. 


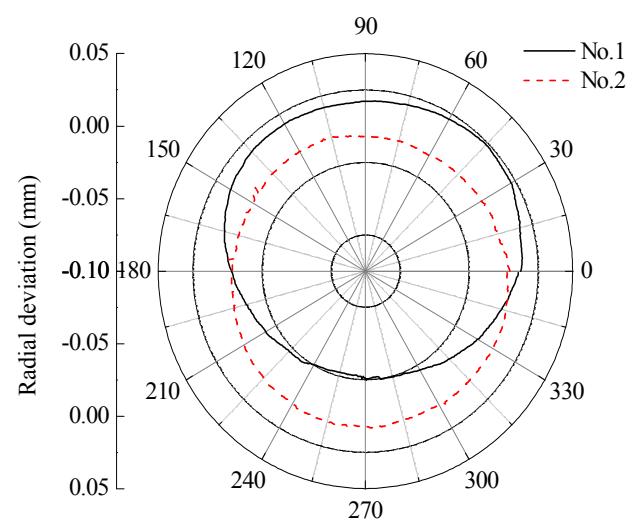

(a) Radial deviation

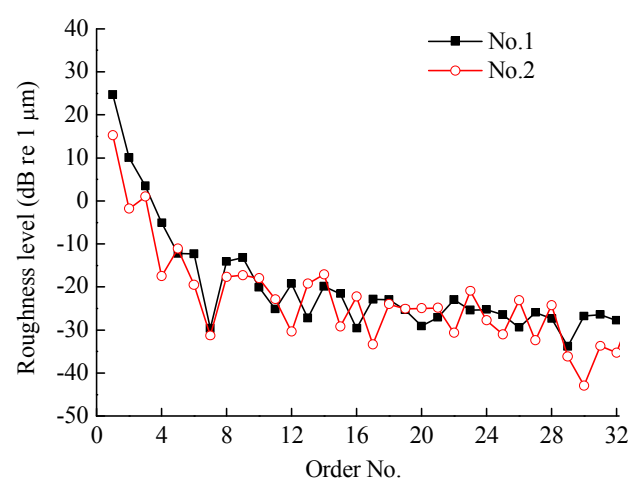

(b) Roughness spectrum

Fig.3 Example of measured out-of-round results for friction rollers

A further key parameter defined as phase position relates the eccentricity of the left and right rollers and forms a primary factor in cut quality. In order to describe this parameter, the phase angle $\varphi$ is used to express the phase position as shown in Fig.4. It can be seen when, $r_{1}$ and $r_{2}$ are defined as the maximum radiuses of the left and right rollers, then $r_{1}^{\prime}$ can be constructed as a mirror image of $r_{1}$ about the vertical direction. The phase angle, $\varphi$ is the defined eccentric angle. In the case of $\varphi$ equals to 0 , the two rollers are predicted as being in phase and the radiuses at the contact points on right and left rollers are equal at that given point in relative rotation. Contrarily, if $\varphi$ is $180^{\circ}$, the two rollers are out of phase. The above cases represent two extreme conditions, the analysis which follows is based on more typical values of phase angle.

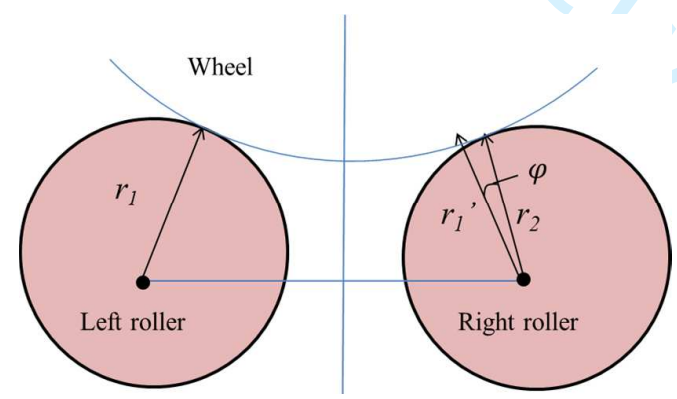

Fig. 4 Definition of phase angle $\varphi$

In the process of cut, the rotational speed of the wheelset is typically around 20rpm. At such a speed, the findings show that any mass eccentricity has no significant impact on the system vibration or cut quality.

\section{Wheel cut modelling}

Based on the preceding discussion, the relationship between the wheel and lathe can be graphically expressed as in 
Fig.5. In the process of wheel cut, the wheel is lifted and driven by two friction rollers. As the clamping devices apply vertical force to the axle boxes, the wheel and two rollers are always in contact. The rotational axis of the friction rollers can be thought to be fixed, with the moving path of the lathe tool controlled by the system's profiling controller. The radius of the wheel being turned in any circumferential position can be determined by the distance between wheelset centre $(\mathrm{O})$ and the lathe tool. The deviation from the mean radius of the wheel can be regarded as the deviation of the centre of the wheel as the lathe tool can be regarded as rigid.

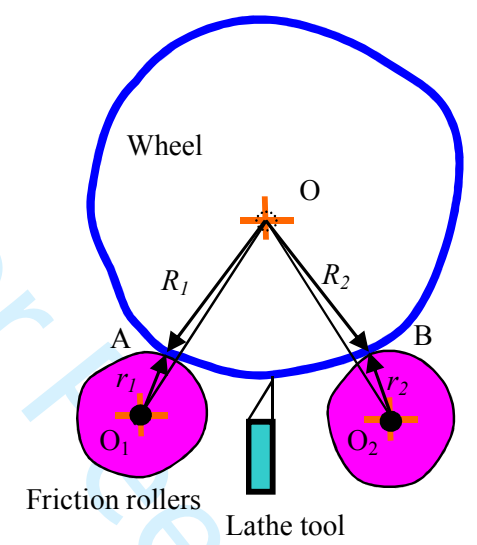

Fig.5 Geometric relationship between wheel, lathe tool and friction rollers

From Fig. 5 it can be seen that under the condition of out-of-round the geometric construction lines connecting the centre of the wheel and rollers cannot pass through the contact points of the wheel and friction rollers. Hence, the coordinate value of wheel centre cannot be obtained by conventional geometric mathematical relations. In this paper, a numerical method is put forward to solve this problem and allow the contact conditions which lead to wheel polyogonisation to be further explored.

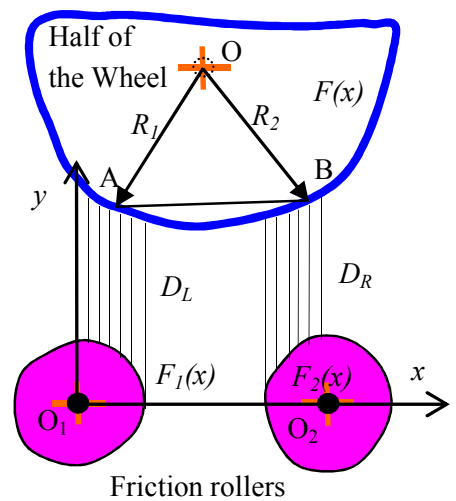

Fig. 6 The diagram used to solve for the contact point between wheel and lathe. 
Establishing the wheel-roller contact points is the first step to calculate the deviation from the mean radius of a wheel. Prior to cut, the deviation of the radius and the mean radius of wheel and rollers can be measured; the coordinate system as shown in Fig.6 is set up. The contour profiles of the two friction rollers can then be expressed as functions $F_{1}(x)$ and $F_{2}(x)$. The wheel is positioned above the rollers with its centre, $O$, placed centrally between the points $O_{1}$ and $\mathrm{O}_{2}$. The wheel contour profile can then be expressed as a function $F(x)$ within the defined coordinate system.

The distance between wheel and rollers can be calculated and the minimum values on the two rollers can be obtained as follows:

$$
D_{L}=\min \left[F(x)-F_{1}(x)\right] ; \quad D_{R}=\min \left[F(x)-F_{2}(x)\right]
$$

$D_{L}$ and $D_{R}$ are the minimum distances between the wheel and the left and right roller,

If $D_{L}$ and $D_{R}$ have similar values, the two corresponding points that have the minimum distance can be considered as the contact points. That can be judged by

$$
\left|D_{L}-D_{R}\right|<\varepsilon
$$

$\varepsilon$ is the allowable error value.

If $D_{L}$ is less than $D_{R}$ but does not satisfy the minimum error criteria of Eq.(4), then the wheel is translated downwards by a distance $D_{L}$, whereby the left roller is now in contact but contact is not achieved with the right roller. Under this condition, the wheel would naturally slide downwards until contact with the right roller is made - with a corresponding change in the contact position of the wheel on the left roller. During these translations, the centre of the wheel $O$ would become offset the middle of $O_{1}$ and $O_{2}$. Hence in the process of searching for the contact points, $D_{L}$ and $D_{R}$ are repeatedly calculated by adjusting the displacement of the wheel $d x$ in the $x$ direction until Eq.(4) is satisfied. Once this is achieved the corresponding contact points on the rollers and the lateral coordinate of wheel centre $x_{o}$ can also be obtained.

The coordinate value of the wheel centre can be computed when the contact points A and B are found through the following equation.

$$
\left\{\begin{array}{l}
\left(x-x_{A}\right)^{2}+\left(y-y_{A}\right)^{2}=R_{1}^{2} \\
\left(x-x_{B}\right)^{2}+\left(y-y_{B}\right)^{2}=R_{2}^{2}
\end{array}\right.
$$

Here, $x_{A}$ and $y_{A}$ are the coordinate values of point contact point A, $x_{B}$ and $y_{B}$ are the coordinate values of contact point $\mathrm{B} . R_{1}$ and $R_{2}$ are the real radius of the wheel at points $\mathrm{A}$ and $\mathrm{B}$.

There are two solutions to Eq.(5) and the correct one can be identified according to Fig.5. It can also be solved using 
the obtained value $x_{o}$ as

$$
y_{0}=-\frac{1}{k} x_{o}+\frac{1}{2}\left(y_{A}+y_{B}\right)+\frac{\left(x_{A}^{2}-x_{B}^{2}\right)}{2\left(y_{A}-y_{B}\right)}-\left(R_{1}^{2}-R_{2}^{2}\right)
$$

Here, $k$ is the slope of the line AB.

\section{Results and discussion}

In the following section, the evolution of wheel polygonization is analysed with respect to the different cut processes and friction rollers eccentricity characteristics discussed above. The simulation requires an accurate radius of wheel and rollers to support the requirement of cut, so the average values of the radius were set to $460 \mathrm{~mm}$ and $110 \mathrm{~mm}$.

\subsection{Number of cuts}

The depth of cut has already been set based on the premeasurement of EMU wheels, however, the number of cuts could be modified to achieve the same depth of cut. The last cut results will have an impact on the relationship between the wheel and friction rollers, so the cut quality will be influenced by the number of cuts. In theory, the more number of cuts, the better cut quality. But the time taken to complete the wheel re-profiling is dependent on the number of cuts being used, adding the number of cuts will decrease the cut efficiency. The optimum number of cuts was analysed based on the calculated results with ideal friction rollers.

Fig. 7 shows the examples of cut results for a wheel with $20^{\text {th }}$ order polygon. The amplitude of the radial deviation is approximately $0.050 \mathrm{~mm}$ before cut, this is reduced to approximately $0.020 \mathrm{~mm}$ after the first cut and further reduced to a level of $0.005 \mathrm{~mm}$ after the second cut. The amplitudes of the radial deviation after the third cut are approximately 0.005 $\mathrm{mm}$ and thus no significantly amplitude reduction was achieved as shown in Fig. 7 (a).

In order to explain this cut result clearly, the roughness level is given in Fig.7 (b). It indicates that the amplitudes of the radial deviations are reduced with the total amount of machining time, which starts from approximately 31.7 $d B$ re $1 \mu m$ before cut. It can be found that the radial deviations are dramatically reduced to $8.3 d B$ re $1 \mu m$ after the first cut, followed by $2.2 d B$ re $1 \mu \mathrm{m}$ after the second cut, and finally, the value is reduced to $0.25 d B$ re $1 \mu m$ after the final (third) cut. The variations of radial deviation are similar between the second and third cuts. Cut results of wheels with different order polygons are also simulated and the roughness levels of the dominant order are picked up as shown in Fig.8. From Fig.8 it can be seen that the first and second cut can reduce the roughness significantly, but roughness levels cannot be improved after the third cut. Operation experience with EMUs has shown that roughness levels around the $20^{\text {th }}$ order neds to be below than $5 d B$ re $1 \mu m$. It can be observed that the result of the second cut has achieved the 
requirements for practical use. Thus, two cuts could be set as the optimum number of cuts to obtain the expected production quality.

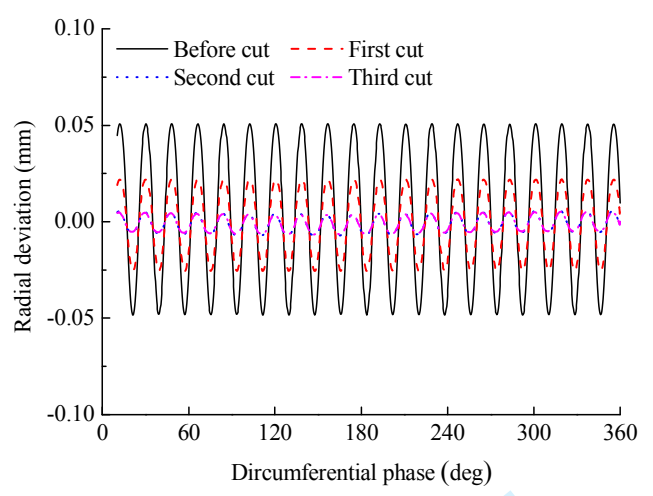

(a)The radial deviation

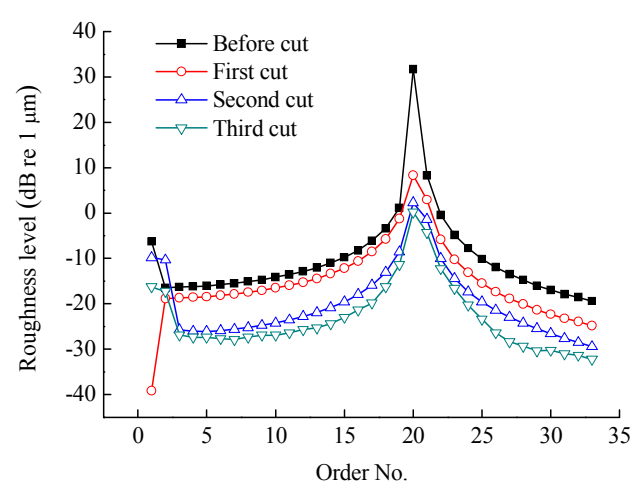

(b)Roughness spectrum

Fig.7 Examples of cut results for a wheel with $20^{\text {th }}$ order polygon

The roughness levels of the dominant order (for example the Order 20 in Fig. 7) are extracted from turned wheels, where each of them has unique defined order from 1 to 20 before cut as shown in Fig.8. It is clear that the first two cuts can evidently reduce the roughness of the wheels with different orders polygon and radius eccentricity, but the third cut has no effective improvement of the wheels' roughness.

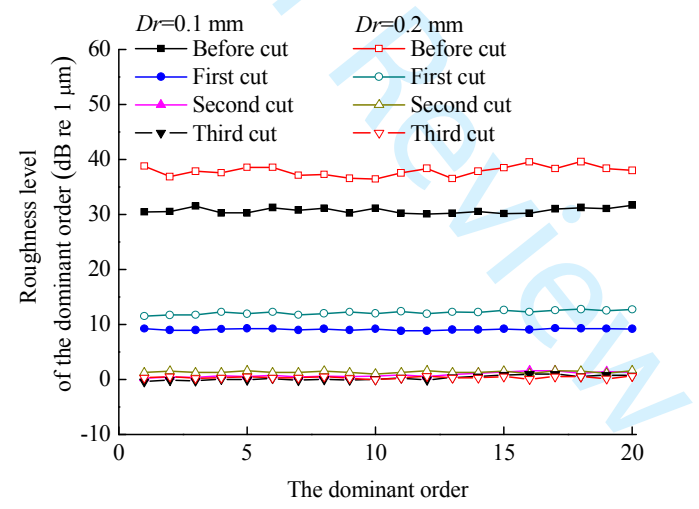

Fig. 8 Cut results of wheels with different order polygons

\subsection{Friction roller eccentricity}

Friction roller eccentricity is a common phenomenon in underfloor wheel lathes due to the inevitable wear of the components, such as the rollers, bearings and transmission system. Once these components exhibit significant wear, the rollers can become eccentric and the quality of cut is then affected accordingly. As the transmission system of each roller operates independently, eccentricity of the rollers is not symmetrical and hence a difference in phase angle $\varphi$ between the rollers occurs, as shown in Fig.4. Based on wheel lathe depot measurements, the phase positions vary from roller to roller, even on the same side of the lathe. In reality, the phase angle $\varphi$ can be adjusted manually to maintain a specific 
value. In this section, the influences that the phase angle $\varphi$ and the eccentricity value on the turning quality are calculated and discussed.

The examples of cut results for wheels with $\varphi$ from 0 to $180^{\circ}$ are shown in Fig.9 to Fig.12. In Fig.9 and Fig.11, the friction roller eccentricity is set $0.1 \mathrm{~mm}$. Wheels with the same phase $(\varphi=0)$ can cause the worst turning quality. This case is investigated and the results are shown in Fig. 9. In this case, the roughness level at the $20^{\text {th }}$ order polygon reduced to $6.4 \mathrm{~dB}$ re $1 \mu \mathrm{m}$ after the first cut, followed by approximately $0.6 \mathrm{~dB}$ re $1 \mu \mathrm{m}$ after the second cut, which is close to the cut results with ideal friction rollers as shown in Fig.7. Fig. 9 also shows that a $4^{\text {th }}$ order polygon can be generated in this case, and the roughness level reached $38.7 d B$ re $1 \mu m$ which exceeds the limiting of $25 d B$ re $1 \mu m$ on EMUs.

Considering the rotation of an eccentric roller, one full rotation will result in the wheel completing one vertical up and down cycle. Furthermore, according to the inherent ratio in radius between the wheel and rollers, four rotations of the rollers are induced during a single complete rotation of the wheel and hence a $4^{\text {th }}$ order polygon can be generated. This phenomenon generally exists in EMU wheels as shown in Fig.10.

The vast majority of significant in-train vibrations and structural damage can be attributed to the high order polygons on the wheels. However, vibration is still generated by the lower order polygons. Additionally, if there are serious low order polygons on wheels, then a high order polygon $\left(20^{\text {th }}\right.$ order in this study) can be initiated and develop readily due to the tracking measurement. It can be summarised that the minimisation of the roughness level is necessary for the process of wheel cut to reduce the risk of unexpected vibration and structural damage.

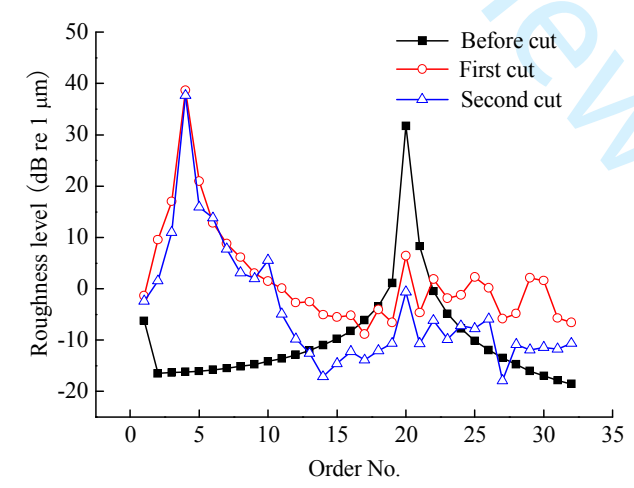

Fig. 9 The results of cut wheels with rollers at the same phase 


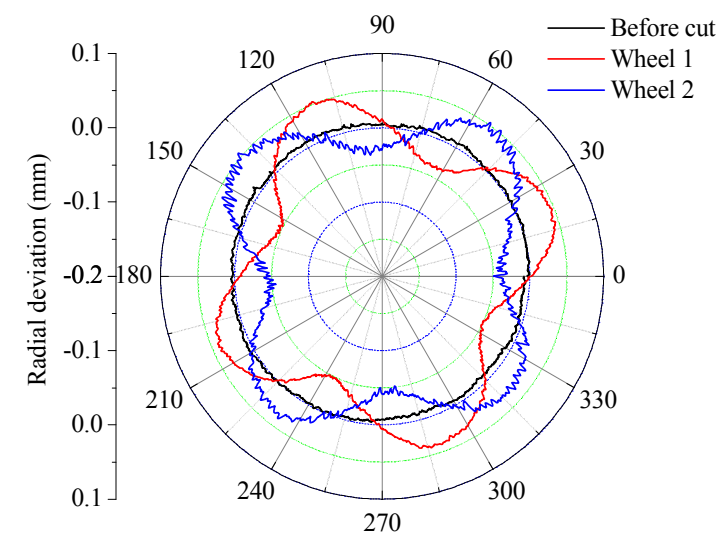

Fig.10 The measured wheels with $4^{\text {th }}$ order polygons

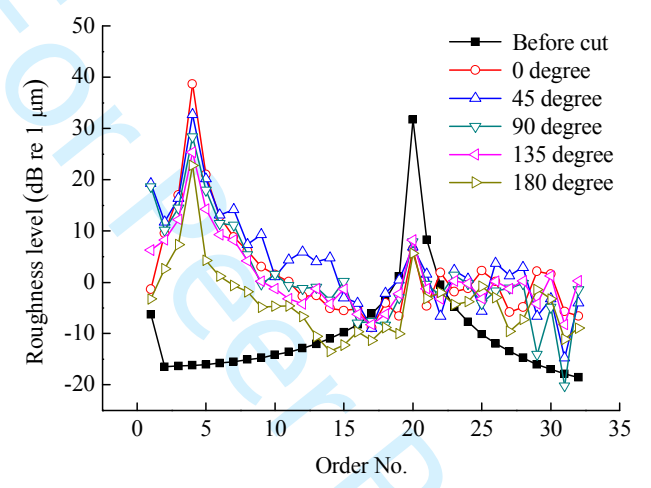

Fig.11 First Cut results of wheels with different phase angles of rollers

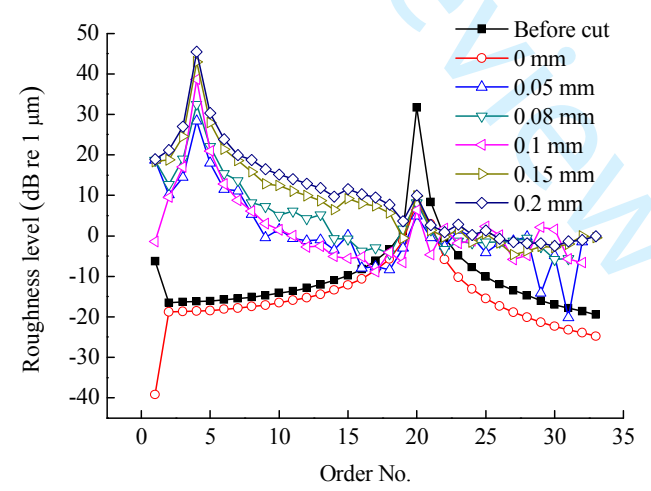

Fig. 12 First Cut results of wheels with different radius eccentricity rollers in the same phase.

In Fig. 9 it can also be seen that the value of the roughness level of the $4^{\text {th }}$ order polygon is slightly changed after the second cut in comparison with the value obtained after the first cut. As a result, from this point onwards only the first cut results with different phase angles of the friction rollers are presented, as shown in Fig. 11. This figure indicates that the phase angle of the rollers does not affect the cut result for higher order polygons, while it greatly influences the magnitude of the $4^{\text {th }}$ order polygon. 
The roughness level of the $4^{\text {th }}$ order polygon is reduced when the phase angles increase from 0 to $180^{\circ}$. This is because the friction rollers with smaller phase angles can cause a larger amplitude of wheel's centre vertical movement. Rollers with a larger radius eccentricity can also lead to larger amplitude vertical movements of the wheel's centre and hence cause more severe $4^{\text {th }}$ order polygons to be formed, as shown in Fig. 12. However, it can also be seen that the level of eccentricity of the friction rollers has an inappreciable effect on the higher order polygons.

\subsection{Spacing of the friction rollers}

As part of the wheel lathe depot test programme, the influence of friction roller spacing on the cut results has been analysed [14]. The work demonstrated that roller spacing variation is not effective in removing wheel low order wheel polyogonisation but can be beneficial in removing higher order polygons. In order to explain this finding in more detail, the influence of the distance between friction rollers is analysed and discussed..

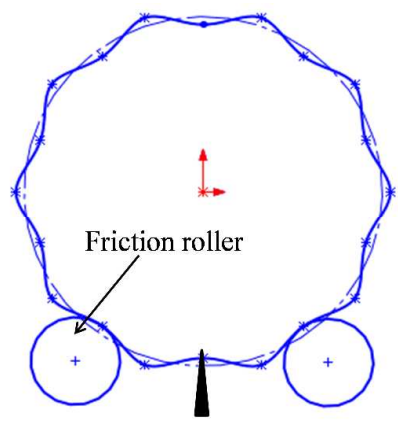

(a)Case 1

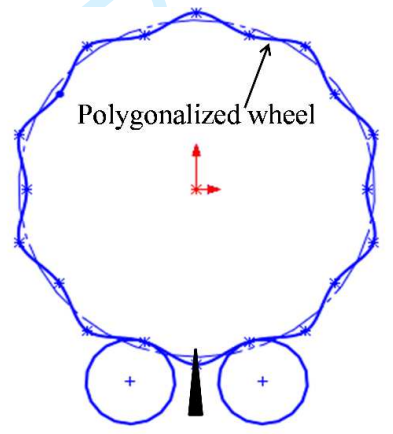

(b) Case 2

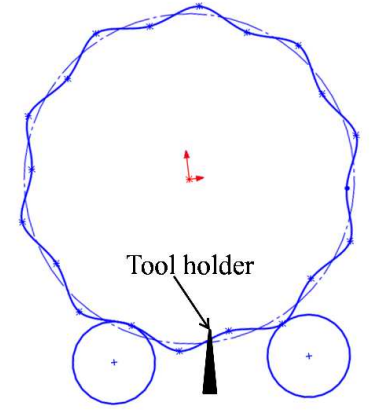

(c) Case 3

Fig.13 Different roller spacing combinations

For a given wheel with a fixed order polygon, variation in spacing of the friction rollers can affect the wheel/rollers contact points and hence the cutting depth of the lathe tool. The influence of different spacing between rollers results in the potential contact combinations illustrated in Fig. 13.

Considering Case 1, the two contacts positions at which the friction rollers contact with the polygonised wheel has the same phase as the contact position at the wheel contact with the tool holder. When the friction rollers drive wheel rotation, the rollers and the tool holder will always contact the crests or troughs of the polygonised wheel at the same time. This condition leads to an almost equal cutting depth around the wheel as compared with wheels that do not exhibit out-of-round. Once the rollers are out-of-round, the radial deviation of the wheel will be added to the eccentricity level of the rollers. This conclusion is proved through calculation as shown in Fig.14. It can be seen that the radial deviation after cut is about $0.1 \mathrm{~mm}$ higher than before, which is corresponds to the radius of eccentricity level of rollers. From Fig. 14(b) 


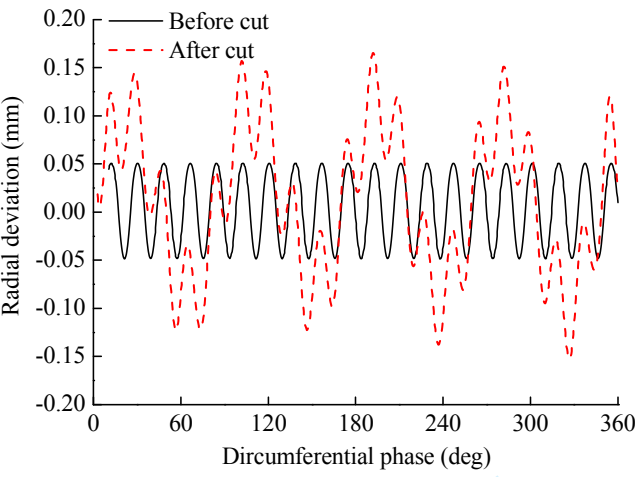

(a)The radial deviation

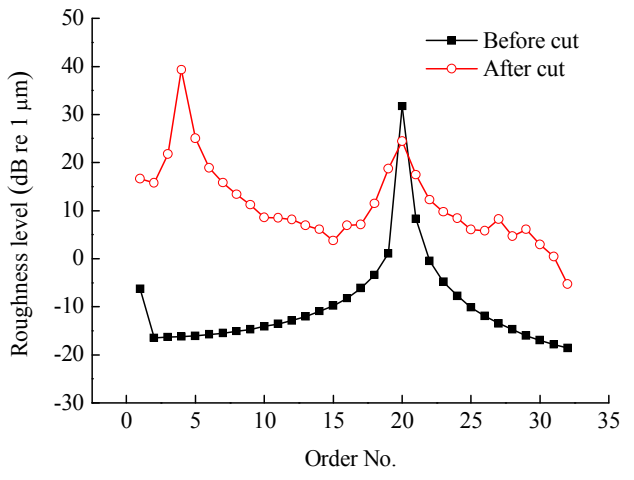

(b)Roughness spectrum

Fig.14 Example cut result (roller spacing, Case 1). (The radius eccentricity value of the rollers is $0.1 \mathrm{~mm}$ and the two rollers have the same phase.)

In case 2, the contact positions at the rollers are in anti-phase with the contact position of the tool holder. When the two friction rollers contact with the wheel at the troughs, the tool holder will contact with the polygonal wheel at its crest. In this condition, the cutting depth is deeper at the crests than that at the troughs. The cut results, in this case, is shown in Fig.15. It can be seen that the result is similar to that of Case 1, however the crests prior to cut are reversed to troughs and vice-versa. This result is due to the cutting depth being too deep at the crests and too low at the troughs.

In Case 3, when one wheel/roller contact point is at the crest of the polygonal wheel, the other contact point is positioned in a trough, and so, the amplitude of the vertical movement of the wheel centre should be smaller. From Fig.16, it can be seen that radius deviation of the wheel after cut is much smaller than that of Case 1 and Case 2, and crucially the high order polyogonisation is effectively removed.

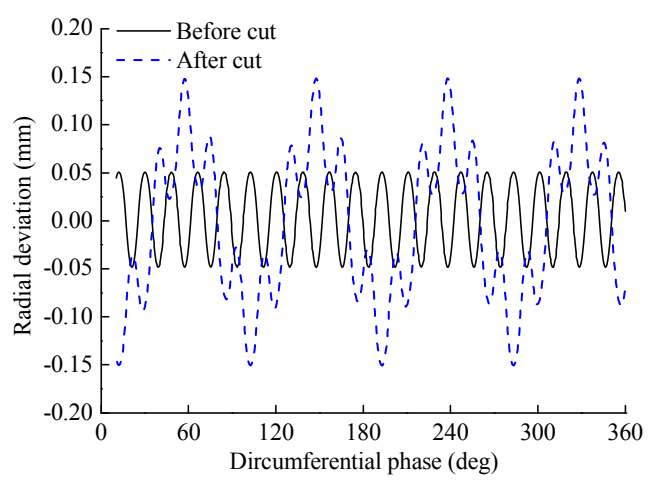

(a)The radial deviation

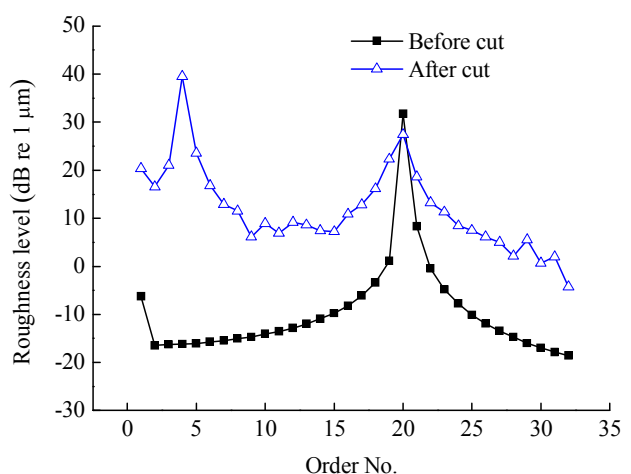

(b)Roughness spectrum

Fig.15 Example cut result (roller spacing, Case 2). The radius eccentricity value of the rollers is $0.1 \mathrm{~mm}$ and the two 
rollers have the same phase.

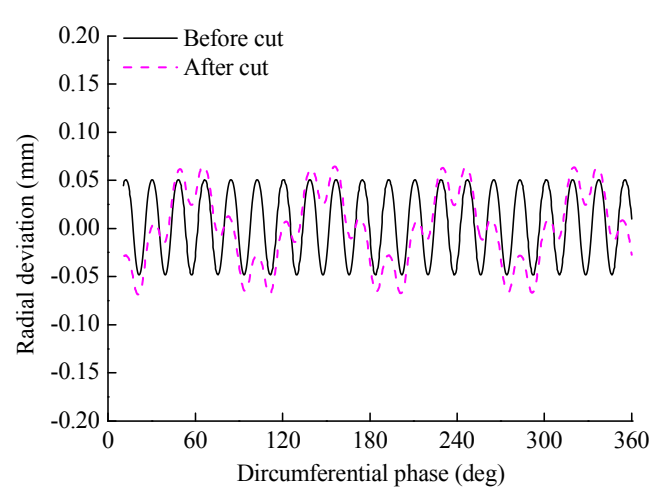

(a)The radial deviation

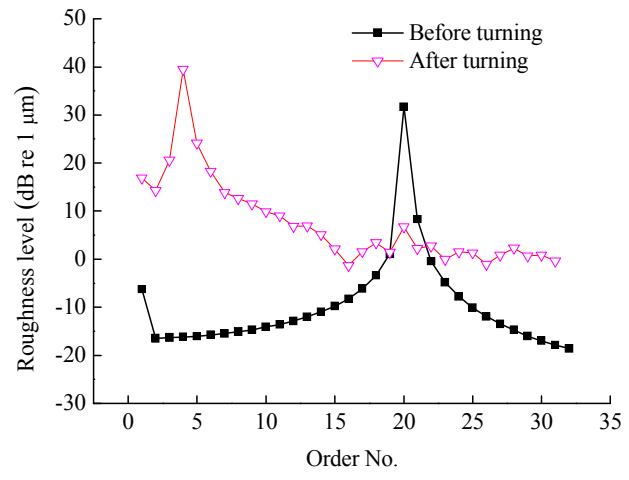

(b)Roughness spectrum

Fig.16 Example cut result (roller spacing, Case 3). The radius eccentricity value of the rollers is $0.1 \mathrm{~mm}$ and the two rollers have the same phase.

\section{Conclusion}

Identification and analysis of high order polygonised wear for Chinese EMU wheels has been presented in this paper. The main wear in form of the friction rollers on existing underfloor lathe was investigated. A numerical model for the wheel cut on the underfloor lathe was built in terms of the correlation between wheels and friction rollers. The effects of the process parameters of the underfloor lathe on wheel polygon were also evaluated.

It was found that at least two cuts were required to remove the high order polygon. The number of cuts needs to be considered based on the time allowed for re-profiling and the cost of re-profiling. The low order polygon appears around the wheel for the eccentricity of the friction rollers. The number of the order depends on the diameter ratio of the wheel to the friction roller. The value and the phase of the eccentricity of the friction rollers have a great impact on the severity of the low order polygon. However, the eccentricity of the friction rollers doesn't affect the cut quality of high order polygon. To achieve removal of high order polygon wheel, the space of two rollers can be adjusted to make the contact points between wheel and rollers separately on the trough and the crest of the polygonal wheel. The optimised method can produce the smallest vibration of the wheel centre whilst obtaining the optimal cut quality.

\section{ACKNOWLEDGEMENT}

The present work was supported by the National Nature Science Foundation of China (Nos.51605394 and U1434201), the Department of Education Foundation of Sichuan Province (No.16ZB0011) and the Key Research Project of Leshan 
City (No.16ZDYJ0147).

\section{References}

[1] Cui Dabin, Li Li, Wang Hengyu, Wen Zefeng, Xiong Jiayang. High-speed EMU wheel re-profiling threshold for complex wear forms from dynamics viewpoint. Wear, 2015, 338-339: 307-315

[2] Regulations of Railway Technical Operation_Part of High-Speed Railway. China Railway. Beijing, 2014.

[3] Ghionea Adrian, Ghionea Ionut, Cioboata Daniela, Savu Mircea. Preliminary considerations regarding modernization of the driving, CNC control and measurement system of a lathe model UBC 150 Rafamet. Scientific Bulletin, Serie C, Fascicle: Mechanics, Tribology, Machine Manufacturing Technology, 2015, 39-46.

[4] Mazilu T., Dumitriu M., Manufacturing technology and repair of railway rolling stock, Matrix Rom, 2013, ISBN 13: 9789737558794

[5] Croitoru M., Contributions to optimize the working set of parameters in cut the elements of rolling stock. PhD. "Gheorghe Asachi" Technical University of Iaşi. 2007.

[6] Han Guangxu, Zhang Jie, Xiao Xinbiao, et al. Study on High-speed Train Abnormal Interior Vibration and Noise Related to Wheel Roughness[J]. Journal of Mechanical Engineering, 2014,50(22):113-121.

[7] Cui Dabin, Liang Shulin, Song Chunyuan, et al. Out of Round High-speed Wheel and Its Influence on Wheel/rail Behavior[J]. Journal of Mechanical Engineering, 2013,49(18):8-16.

[8] Nielsen J C O, Johansson A. Out-of-round railway wheels-A literature survey[J]. IMechE Journal of Rail and Rapid Transit, 2000, 214(F):79-91.

[9] Chen Wei, Dai Huan-yun, LUO Ren. Effect of High Order Polygons of Wheels for High Speed Trains on Dynamics Performance of Vehicles[J]. Rolling Stock, 2014,52(12):4-8.

[10] Brake W, Chiu W.A review of the effects of out-of-round wheels on track and vehicle components[J], IMechE Journal of Rail and Rapid Transit,2005:291,151-175.

[11] Johansson A., Andersson C.. Out-of-round railway wheels-a study of wheel polygonalization through simulation of three-dimensional wheel-rail interaction and wear, Vehicle System Dynamics. 43(8): 539-559,2005.

[12] Huang N E, Shen S S P. Hilbert-Huang transform and its applications[M]. USA: World Scientific Publishing Co. Pte. Ltd. 2005.

[13] Su Jian, Li, Li, Cui Dabin. Study on influence of turning repair operations on wheels with initial polygonal state. Journal of the China Railway Society. 2017, 39(5):38-61.

[14] Tao Gongquan, Wang Linfeng, Wen Zefeng, Guan Qinghua, Jin Xuesong. Measurement and assessment of 
out-of-round electric locomotive wheels. Proc IMechE Part F: J Rail and Rapid Transit. 2018, 232:275-287. 